\title{
Multiple Peer-To-Peer Relay Network Robust Beamforming Based on Lorentz-Positive Matrices
}

\author{
Jianhua $\mathrm{Li}^{1}$ and Yunshan $\mathrm{Hou}^{2 *}$ \\ ${ }^{1}$ College of Mathematical Science, Luoyang Normal University, Luoyang, 471022, \\ China; \\ ${ }^{2}$ College of Information Engineering, Henan University of Science and \\ Technology, Luoyang, 471023, China \\ 122406735@qq.com, ${ }^{2}$ houys20134@163.com
}

\begin{abstract}
Aiming at minimizing the total transmit power of relay nodes in the multiple peer-topeer relay network in the presence of channel estimation error, a relay beamforming method based on Lorentz positive matrix is proposed. Firstly, assuming the channel state information of the second hop in the relay network is uncertain and the signal to noise ratios of the destination nodes are given, the expression of total relay nodes transmit power minimization optimization problem is deduced. Secondly, some real valued variables are defined and the concept of Lorentz cone is used to transform the optimization problem into a real valued problem. Finally, using the equivalent linear matrix inequality description of Lorentz positive matrices, the relay transmit power minimization problem is transformed into a real valued convex optimization problem. The proposed method overcomes the problem existing in the conventional robust relay beamforming methods based on semi definite relaxation technique, which sometimes yield solution matrix of with a rank greater than 1. Simulation results show that in the case of different relay nodes and channel error coefficients, the proposed method yields minimum relay transmit power in line with the results of the robust relay beamforming method based on semi definite relaxation technique, which validates the effectiveness of the proposed method.
\end{abstract}

Keywords: relay network; robust beamforming; channel state information; transmit power; semi-definite relaxation

\section{Introduction}

In recent years, collaborative relay beamforming algorithms for wireless communication networks with perfect channel state information(CSI) has been well studied to enhance the utilization of the network resources [1-6]. In these networks, multiple source-destination pairs (peer-to-peer) typically share a channel.

For instance, a method based on semidefinite programming relaxation was proposed to calculate the distributed relay beamforming weights in [7]. To minimize the total transmit power of the network subject to quality-of-service(Qos) constraints at each destination, the problem of joint source power control and general rank relay matrix design was solved in [8].

However, since in practice estimation errors and quantization errors in CSI are inevitable, they should be considered in system design. One approach is to consider the worst-case based optimization that restricts the channel errors in a certain bounded region [9-10]. Another approach is the probabilistic approach, in which QoS is guaranteed in a probabilistic sense [11-13].

${ }^{*}$ Corresponding Author 
The first approach usually employs semi-definite programming relaxation to tackle the problem of infinite constraints due to CSI errors [14], which can not guarantee a rank-one optimal solution. In the case of solution with a general rank, a randomization procedure has to be utilized to approximate a rank-1 matrix.

In order to overcome this disadvantage, a method based on real value Lorentz-positive maps and linear matrix inequalities is proposed, which converts the transmit power minimization problem at relay nodes into a convex optimization problem, which can then be solved efficiently with convex software. Specifically, we model channel estimation error of the second hop as a Gaussian random vector with known statistical distribution. The objective is to minimize the total transmit power at relays subject to QoS constraints at each receiver.

\section{Problem Formulation}

Consider a network where $K$ source(S)-destination(D) pairs communicate through a set of $M$ distributed relays $\left\{R_{i}\right\}$, as shown in Fig. 1. Each node in the network is assumed to have a single antenna, and there is no direct link between each $\left(S_{k}, D_{k}\right)$ pair. We denote source- $k$ and destination- $k$ by $S_{k}$ and $D_{k}$, respectively. The received signal in the first time slot at $R_{i}$ is

$x_{i}=\sum_{k=1}^{K} g_{k i} s_{k}+n_{i}$

where $s_{k}$ is the data symbol transmitted from $S_{k}, g_{k i}$ is the Rayleigh flat fading channel from $S_{k}$ to $R_{i}$, and $n_{i}$ is the zero mean circularly symmetric Gaussian noise at each $R_{i}$ with variance $\sigma_{R}^{2}$. Without loss of generality, we assume that $E\left\{s_{k}\right\}=0, E\left\{\left|s_{k}\right|^{2}\right\}=p_{k}$, and different $s_{k}$ 's are independent from each other. Due to the distributed nature, each $R_{i}$ multiplies the received signal by a scalar weight $w_{i}$ and then forwards it to the destination.

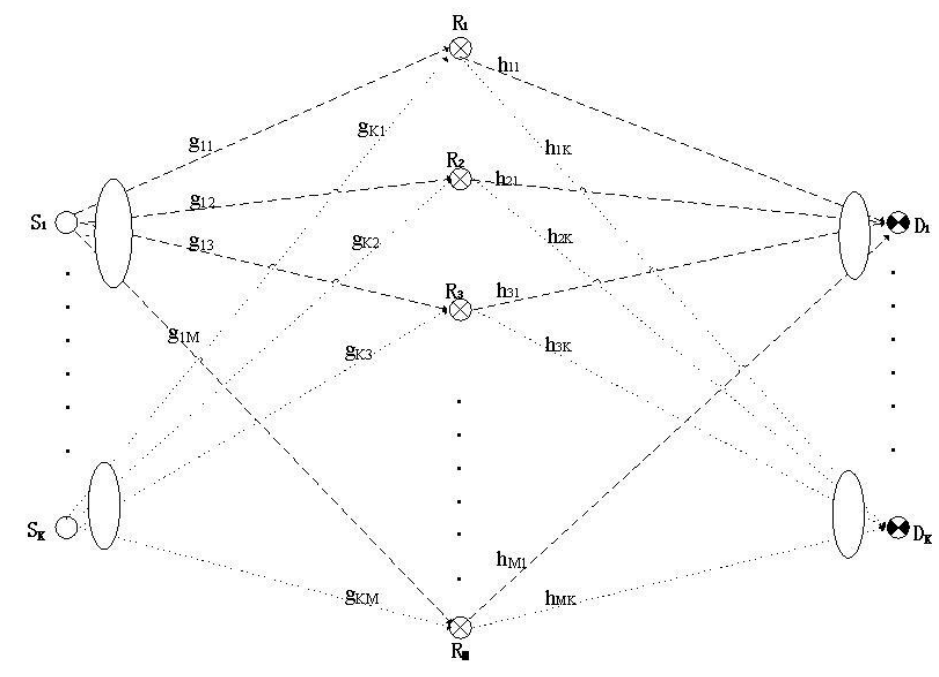

Figure 1. A Relay Network with K Source-Destination Pairs and M Relays

The signal received at $D_{k}$ in the second time slot is 


$$
\begin{aligned}
& y_{k}=\sum_{i=1}^{M} h_{i k} w_{i} x_{i}+z_{k} \\
& =\sum_{i=1}^{M} h_{i k} w_{i}\left(\sum_{k=1}^{K} g_{k i} s_{k}+n_{i}\right)+z_{k} \\
& =\sum_{i=1}^{M} h_{i k} w_{i} g_{k i} s_{k}+\sum_{i=1}^{M} h_{i k} w_{i} \sum_{\substack{l=1 \\
l \neq k}}^{K} g_{l i} s_{l}+\sum_{i=1}^{M} h_{i k} w_{i} n_{i}+z_{k}
\end{aligned}
$$

where $h_{i k}$ is the Rayleigh flat-fading channel from $R_{i}$ to $D_{k}$, and $z_{k} \sim C N\left(0, \sigma_{D}^{2}\right)$ is the noise at $D_{k}$. Note that independence among channels $\left\{g_{l i}\right\}$ and $\left\{h_{i k}\right\}$ is also assumed. The SINR at $D_{k}$ can be expressed as

$$
\operatorname{SINR}_{k}=\frac{\left|\sum_{i=1}^{M} h_{i k} w_{i} g_{k i}\right|^{2} p_{k}}{\sum_{\substack{l=1 \\ l \neq k}}^{K}\left|\sum_{i=1}^{M} h_{i k} w_{i} g_{l i}\right|^{2} p_{l}+\sum_{i=1}^{M}\left|h_{i k} w_{i}\right|^{2} \sigma_{R}^{2}+\sigma_{D}^{2}}
$$

The average total power transmitted at relays is

$P_{T}=\sum_{i=1}^{M}\left|w_{i}\right|^{2}\left(\sum_{k=1}^{K}\left|g_{k i}\right|^{2} p_{k}+\sigma_{R}^{2}\right)$

The optimization problem with perfect CSI can be mathematically formulated as

$\min _{\left\{w_{i}\right\}} P_{T}$

s.t. $\quad \operatorname{SINR}_{k} \geq \gamma_{k}, \quad k=1,2, \cdots, K$

where $\gamma_{k}$ is the predefined threshold. The solution to (5) has been developed in [2] by applying semidefinite relaxation technique.

Since channels $\left\{g_{k i}\right\}$ can be directly estimated at each $R_{i}$, and knowledge of channels $\left\{h_{i k}\right\}$ at $R_{i}$ depends upon the feedback from $\left\{D_{k}\right\}$, the level of uncertainty is much higher in the $R_{i}$ to $D_{k}$ links. To account for this imperfection, the channel knowledge available at each relay is modeled as $\mathbf{h}_{k}=\overline{\mathbf{h}}_{k}+\boldsymbol{\delta}_{k}$, where $\mathbf{h}_{k}=\left[h_{1 k}, h_{2 k}, \cdots, h_{M k}\right]^{\mathrm{H}}, \overline{\mathbf{h}}_{k}$ is the estimate of $\mathbf{h}_{k}$, and $\left\|\boldsymbol{\delta}_{k}\right\| \leq \varepsilon_{k}$ is the corresponding error.

For convenience, let us introduce variables in vector notation,

$G_{k}=\sqrt{p_{k}} \operatorname{diag}\left(\left[g_{k 1}, g_{k 2}, \cdots, g_{k M}\right]^{\mathrm{T}}\right)$

$\mathbf{w}=\left[w_{1}, w_{2}, \cdots, w_{M}\right]^{\mathrm{T}}$

$\mathbf{v}_{k}=G_{k} \mathbf{w}$ 
$\tilde{\mathbf{w}}_{i}=\left[0, \cdots, 0, w_{i}, 0, \cdots, 0\right]^{\mathrm{T}},(i=1,2, \cdots, M)$

Therefore (3) is equivalent to

$$
\operatorname{SINR}_{k}=\frac{\left|\mathbf{h}_{k}^{\mathrm{H}} \mathbf{v}_{k}\right|^{2}}{\sum_{l=1, l \neq k}^{L}\left|\mathbf{h}_{k}^{\mathrm{H}} \mathbf{v}_{l}\right|^{2}+\sigma_{R}^{2} \sum_{i=1}^{M}\left|\mathbf{h}_{k}^{\mathrm{H}} \tilde{\mathbf{w}}_{i}\right|^{2}+\sigma_{D}^{2}}
$$

With imperfect CSI, the optimization problem is expressed as

$\min _{\left\{w_{i}\right\}} P_{T}$

s.t. $\frac{\left|\left(\overline{\mathbf{h}}_{k}+\boldsymbol{\delta}_{k}\right)^{\mathrm{H}} \mathbf{v}_{k}\right|^{2}}{\sum_{l=1, l \neq k}^{L}\left|\left(\overline{\mathbf{h}}_{k}+\boldsymbol{\delta}_{k}\right)^{\mathrm{H}} \mathbf{v}_{l}\right|^{2}+\sigma_{R}^{2} \sum_{i=1}^{M}\left|\left(\overline{\mathbf{h}}_{k}+\boldsymbol{\delta}_{k}\right)^{\mathrm{H}} \tilde{\mathbf{w}}_{i}\right|^{2}+\sigma_{D}^{2}} \geq \gamma_{k}$

$\forall \boldsymbol{\delta}_{k}:\left\|\boldsymbol{\delta}_{k}\right\| \leq \varepsilon_{k}, k=1,2, \cdots, K$

A more conservative form of (8) is

$\min _{\left\{w_{i}\right\}} P_{T}$

s.t. $\frac{1}{\sqrt{\gamma_{k}}} \operatorname{Re}\left\{\left(\overline{\mathbf{h}}_{k}+\boldsymbol{\delta}_{k}\right)^{\mathrm{H}} \mathbf{v}_{k}\right\}$

$\geq \sqrt{\sum_{l=1, l \neq k}^{L}\left|\left(\overline{\mathbf{h}}_{k}+\boldsymbol{\delta}_{k}\right)^{\mathrm{H}} \mathbf{v}_{l}\right|^{2}+\sigma_{R}^{2} \sum_{i=1}^{M}\left|\left(\overline{\mathbf{h}}_{k}+\boldsymbol{\delta}_{k}\right)^{\mathrm{H}} \tilde{\mathbf{w}}_{i}\right|^{2}+\sigma_{D}^{2}}$

$\forall \boldsymbol{\delta}_{k}:\left\|\boldsymbol{\delta}_{k}\right\| \leq \varepsilon_{k}, k=1,2, \cdots, K$

\section{Robust Optimization Based on Lorentz-Positive Maps}

First we rewrite (9) into a problem with real-valued design variables. We write $\mathbf{w}$ as $\mathbf{w}=\mathbf{w}_{1}+j \mathbf{w}_{2}$, where $\mathbf{w}_{1}=\operatorname{real}(\mathbf{w})$ is the real part of $\mathbf{w}, \mathbf{w}_{2}=\operatorname{imag}(\mathbf{w})$ is the imaginary part of $\mathbf{w}$. Obviously $\mathbf{w}_{1} \in R^{M}$ and $\mathbf{w}_{2} \in R^{M}$. Likewise, we define $\overline{\mathbf{h}}_{k}=\overline{\mathbf{h}}_{k 1}+j \overline{\mathbf{h}}_{k 2}, \boldsymbol{\delta}_{k}=\boldsymbol{\delta}_{k 1}+j \boldsymbol{\delta}_{k 2} . \tilde{\mathbf{w}}_{k}=\tilde{\mathbf{w}}_{k 1}+j \tilde{\mathbf{w}}_{k 2}, \mathbf{v}_{k}=\mathbf{v}_{k 1}+j \mathbf{v}_{k 2},(k=1,2, \cdots, M)$.

Let's define $\mathbf{V}_{-k, 1}=\left[\mathbf{v}_{11}, \cdots, \mathbf{v}_{k-1,1}, \mathbf{v}_{k+1,1}, \cdots, \mathbf{v}_{K 1}\right]$,

$\mathbf{V}_{-k, 2}=\left[\mathbf{v}_{12}, \cdots, \mathbf{v}_{k-1,2}, \mathbf{v}_{k+1,2}, \cdots, \mathbf{v}_{K 2}\right]$,

$\tilde{\mathbf{W}}_{1}=\left[\tilde{\mathbf{w}}_{11}, \tilde{\mathbf{w}}_{21}, \cdots, \tilde{\mathbf{w}}_{M 1}\right], \quad \tilde{\mathbf{W}}_{2}=\left[\tilde{\mathbf{w}}_{12}, \tilde{\mathbf{w}}_{22}, \cdots, \tilde{\mathbf{w}}_{M 2}\right]$, 


$$
\begin{aligned}
\mathbf{C}_{k}^{\mathrm{T}} & =\left[\begin{array}{llllll}
\frac{1}{\sqrt{\gamma_{k}}} \mathbf{V}_{k 1} & \mathbf{V}_{-k, 1} & \mathbf{V}_{-k, 2} & \sigma_{R} \tilde{\mathbf{W}}_{1} & \sigma_{R} \tilde{\mathbf{W}}_{2} & 0 \\
\frac{1}{\sqrt{\gamma_{k}}} \mathbf{V}_{k 2} & \mathbf{V}_{-k, 2} & -\mathbf{V}_{-k, 1} & \sigma_{R} \tilde{\mathbf{W}}_{2} & -\sigma_{R} \tilde{\mathbf{W}}_{1} & 0
\end{array}\right] \in R^{2 M \times 2(M+K)}, \\
\mathbf{c}_{k}^{\mathrm{T}} & =\left[\begin{array}{ll}
\overline{\mathbf{h}}_{k 1}^{\mathrm{T}} & \overline{\mathbf{h}}_{k 2}^{\mathrm{T}}
\end{array}\right] \mathbf{C}_{k}^{\mathrm{T}}+\left[0, \cdots, 0, \sigma_{D}\right] \in R^{1 \times 2(M+L)} .
\end{aligned}
$$

Thus (9) can be expressed as the following real-valued problem:

$$
\min _{\left\{w_{i}\right\}} P_{T}
$$

s.t. $\quad \mathbf{C}_{k}\left[\begin{array}{l}\boldsymbol{\delta}_{k 1} \\ \boldsymbol{\delta}_{k 2}\end{array}\right]+\mathbf{c}_{k} \in L^{2(M+K)}$

$\forall\left\|\left[\begin{array}{c}\boldsymbol{\delta}_{k 1} \\ \boldsymbol{\delta}_{k 2}\end{array}\right]\right\| \leq \varepsilon_{k}, k=1,2, \cdots, K$

where $L^{K}$ represents $K$-dimensional Lorentz cone, for an arbitrary

$\mathbf{x}=\left(x_{1}, \cdots, x_{K}\right) \in R^{K}$, it is defined as $L^{K}=\left\{\mathbf{x} \in R^{K} \mid x_{1} \geq \sqrt{x_{2}^{2}+\cdots+x_{K}^{2}}\right\}$.

Define $\mathbf{B}_{k}=\left[\begin{array}{ll}\mathbf{c}_{k} & \boldsymbol{\varepsilon}_{k} \mathbf{C}_{k}\end{array}\right], \quad \tilde{\boldsymbol{\delta}}_{k}=\left[1, \boldsymbol{\delta}_{k 1}^{\mathrm{T}}, \boldsymbol{\delta}_{k 2}^{\mathrm{T}}\right]$, we obtain the equivalent expression of (9) as

$\min _{\left\{w_{i}\right\}} P_{T}$

s.t. $\quad \mathbf{B}_{k} \tilde{\boldsymbol{\delta}}_{k} \in L^{2(M+K)}, \forall \tilde{\boldsymbol{\delta}}_{k} \in L^{2 M+1}, \quad k=1,2, \cdots, K$

In order to solve (11), we define the following set

$\mathrm{B}=\left\{\mathbf{B}_{k} \in L^{2(M+K) \times(2 M+1)} \mid \mathbf{B}_{k} \mathbf{y}_{k} \in L^{2(M+K)}, \forall \mathbf{y}_{k} \in L^{2 M+1}\right\}$

From reference [9] we know the set $\mathrm{B}$ in (12) is equivalent to the matrix $\mathbf{B}_{k}$ being Lorentz-positive, namely

$$
\mathbf{x}_{k}^{T} \mathbf{B}_{k} \mathbf{y}_{k} \geq 0, \forall \mathbf{x}_{k} \in L^{2(M+K)}, \forall \mathbf{y}_{k} \in L^{2 M+1}
$$

The set B in (12) of all Lorentz-positive matrices forms a closed convex cone, which has an LMI description.

Furthermore, (13) is equivalent to the existence of $\mathbf{X}_{k} \in \ell_{2(M+K)-1,2 M}^{\perp}$ which satisfies ${ }^{[15]}$

$\hat{A}\left(\mathbf{B}_{k}\right)+\mathbf{X}_{k} \in \mathrm{S}_{+}^{(2(M+K)-1) * 2 M}$

Where $S_{+}^{N}$ denotes $N \times N$ positive semidefinite symmetric matrices, $\ell_{N, M}^{\perp}$ stands for $N M \times N M$ symmetric matrices, which consist of $N \times N$ skew-symmetric blocks of dimension $M \times M . \hat{A}(\mathbf{G})$ refers to the arrow matrix of $\mathbf{G}$, which is defined as 


$$
\hat{\mathbf{A}}(\mathbf{G})=\left[\begin{array}{ccccc}
A\left(\mathbf{g}_{0}\right) & A\left(\mathbf{g}_{3}\right) & A\left(\mathbf{g}_{4}\right) & \cdots & A\left(\mathbf{g}_{L}\right) \\
A\left(\mathbf{g}_{3}\right) & A\left(\mathbf{g}_{-1}\right) & 0 & \cdots & 0 \\
A\left(\mathbf{g}_{4}\right) & 0 & A\left(\mathbf{g}_{-1}\right) & \cdots & 0 \\
\vdots & \vdots & \vdots & \ddots & \vdots \\
A\left(\mathbf{g}_{L}\right) & 0 & 0 & \cdots & A\left(\mathbf{g}_{-1}\right)
\end{array}\right]
$$

where $\mathbf{g}_{l}$ denote the $l$-th row of $\boldsymbol{G}$, and $\boldsymbol{g}_{0}=\boldsymbol{g}_{1}+\boldsymbol{g}_{2}$, and $\boldsymbol{g}_{-1}=\boldsymbol{g}_{1}-\boldsymbol{g}_{2}$.

In formula (15), the matrix $\boldsymbol{A}(\boldsymbol{a})$ is generated by a vector $\boldsymbol{a}$. Assuming $\boldsymbol{a}=\left[a_{1}, a_{2}, \ldots, a_{K}\right]^{T}$ (with $K \geq 3$ ), $\boldsymbol{A}(\boldsymbol{a})$ is expressed as

$$
\boldsymbol{A}(\boldsymbol{a})=\left[\begin{array}{ccccc}
a_{1}+a_{2} & a_{3} & a_{4} & \cdots & a_{K} \\
a_{3} & a_{1}-a_{2} & 0 & \cdots & 0 \\
a_{4} & 0 & a_{1}-a_{2} & \cdots & 0 \\
\vdots & \vdots & \vdots & \ddots & \vdots \\
a_{K} & 0 & 0 & \cdots & a_{1}-a_{2}
\end{array}\right]
$$

In view of the above formulas, the optimization problem in (11) is finally converted to

$$
\begin{array}{ll}
\min _{\left\{w_{i}\right\}} & P_{T} \\
\text { s.t. } & \hat{A}\left(\mathbf{B}_{k}\right)+\mathbf{X}_{k} \geq \mathbf{0}, \quad k=1,2, \cdots, K \\
\mathbf{X}_{k} \in \ell_{2(M+K)-1,2 M}^{\perp}
\end{array}
$$

\section{Numerical Results}

In this section, we numerically examine the performance of our proposed robust beamforming design. The method in [9] is named as SDR (Semi-Definite Relaxation) , our proposed method shown in (17) is named as LPM(Lorentz-Positive Map). In the simulations, we compare the performance of these two methods as well as with the perfect CSI case. All results are averaged over 1000 Monte Carlo simulation runs.

In all examples, the parameters are set as, $K=3, \quad M=8, \sigma_{R}^{2}=\sigma_{D}^{2}=0.1$. The norm of all channel estimates is assumed to be one, namely $\left\|\overline{\mathbf{h}}_{k}\right\|^{2}=1$, and we assume $p_{k}=1, \quad(k=1,2, \cdots, K)$, error vector $\varepsilon_{k}=\varepsilon\left\|\overline{\mathbf{h}}_{k}\right\|$.

Figure 2 shows the problem feasibility rate versus the relative perturbation bound $\varepsilon(\varepsilon \in\{0.01,0.04,0.07,0.10\})$. As we can see, the feasibility rate of the LPM problem is only slightly lower than that of SDP relaxation problem, and this behavior coincides with the fact that (9) is a more conservative (but convex) form than (8), but not excessively so. It is observed that the feasibility rate decreases when the perturbation bound increases, and that the feasibility rate increases when the number of relay nodes increases. 

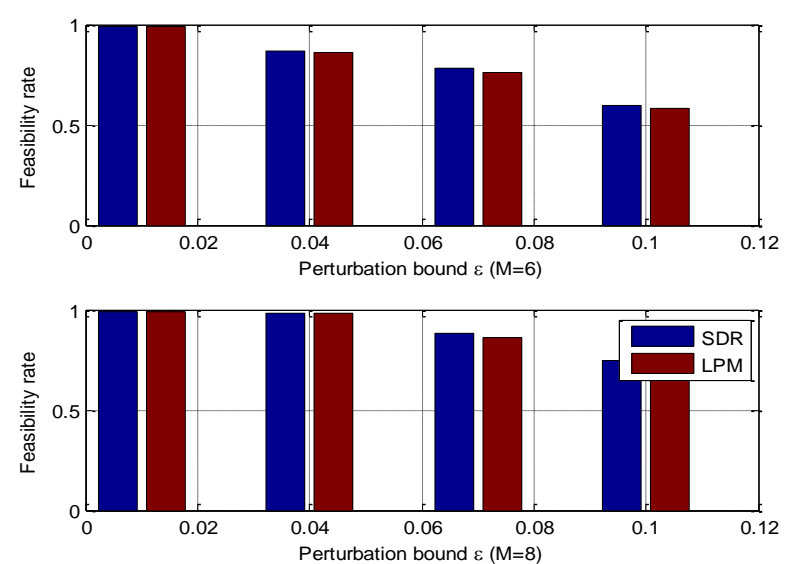

Figure 2. Feasibility Rate vs. the Perturbation Bound $\varepsilon$

Figure 3, displays the average total transmission power of relay nodes versus the perturbation bound. As expected, the higher transmission power is required to meet the robust QoS constraints for the larger bound of uncertainty, as well as for less transmit antennas. From the simulations results, it is clearly seen that the more conservative model in (9) is sufficiently tight in practice.

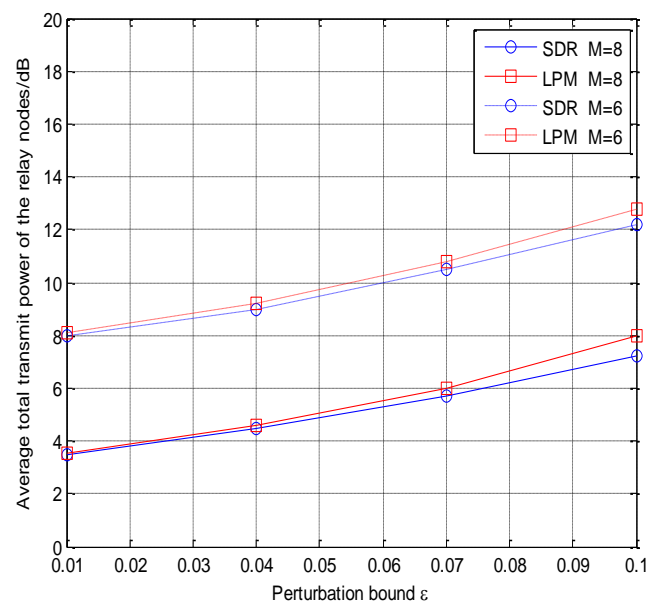

Figure 3. Average Total Transmit Power of Relay Nodes vs. the Perturbation Bound $\varepsilon$

In Figure 4, and Figure 5, we plot the minimum average total transmit power of the relay nodes versus the target SINR with $M=8$ for different values of $\varepsilon=0.01$ and $\varepsilon=0.04$. In both figures it can be seen that more transmit power is needed when the required SINR at the destination nodes is higher, which conforms to our common sense. To meet the same SINR requirement at the destination nodes, LPM needs a little more transmit power than SDR, which is rather negligible. 


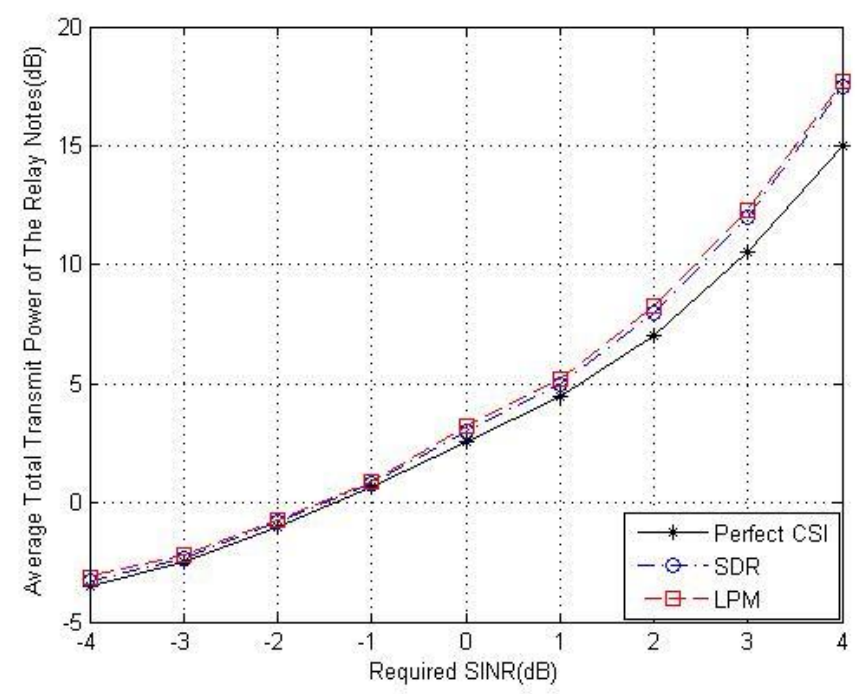

Figure 4. Average Total Transmit Power of the Relay Nodes Versus the Target SINR

$(\varepsilon=0.01, M=8)$

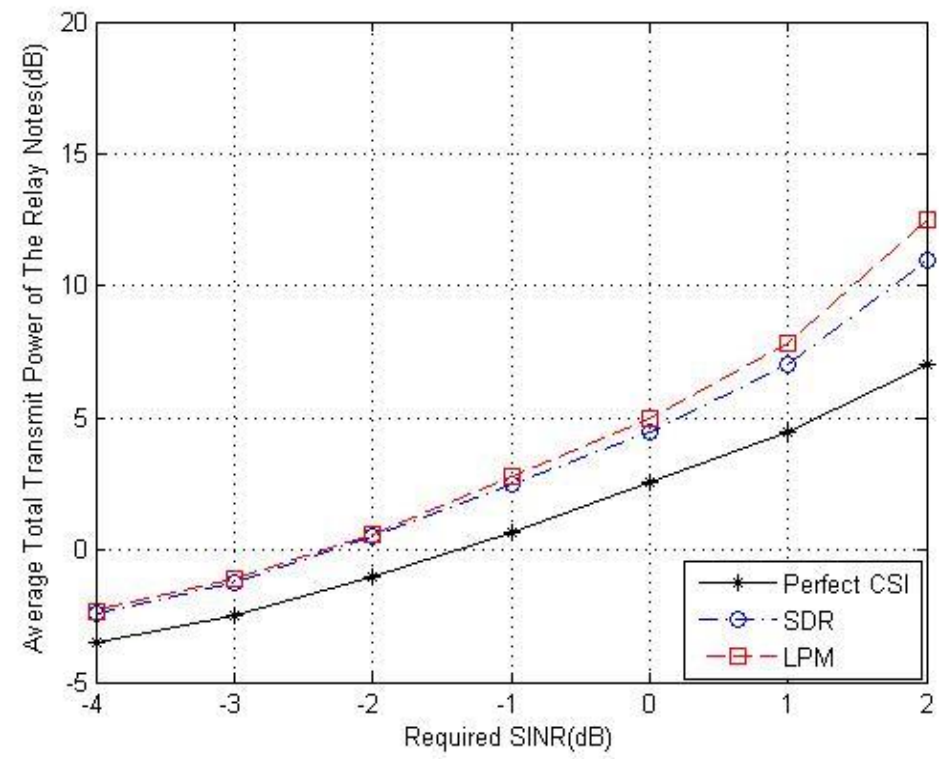

Figure 5. Average Total Transmit Power of the Relay Nodes Versus the Target SINR

$(\varepsilon=0.04, M=8)$

\section{Conclusions}

This paper proposes a robust beamforming method for multiple peer-to-peer relay networks. This method solves the transmit power minimization problem at relay nodes subject to the quality of service at each receiver in the context of imperfect channel state information at the relays. The proposed method is based on real value Lorentz-positive maps and linear matrix inequalities, which converts the transmit power minimization problem at relay nodes into a convex optimization problem. The proposed method 
overcomes the disadvantages of traditional methods based on semi-definite programming relaxation, which sometimes result in solutions with rank greater than one. Simulation results show that the solutions of the proposed method are consistent with those of conventional method based on semi-definite programming relaxation.

\section{Acknowledgments}

This study was partially supported by the National natural science foundation of China under grant No. U1404615 and the natural science foundation of Henan province in China under grant No. 142300410343, 162400410122 and Key research projects in Henan province colleges and universities (15B120005).

\section{References}

[1] W. Chao, W. H. Ming and N. Derrick and W. Kwan, "Joint beamforming and power allocation for secrecy in peer-to-peer relay networks", IEEE Transactions on Wireless Communications, vol. 14, no. 6, (2015), pp. 3280-3293.

[2] A.Ali, M. N. N. Telex and A. D. Octavia, "Cooperative AF Relaying With Beamforming and Limited Feedback in Cognitive Radio Networks”, IEEE Communications Letters, vol. 19, no. 3, (2015), pp. 491494.

[3] C. C. Chen, C. S. Tseng and D.Juwendo, "Adaptive distributed beamforming for amplify-and-forward relay networks: Convergence analysis", IEEE Transactions on Wireless Communications, vol. 13, no. 8, (2014), pp. 4167-4178.

[4] M. Behrad and M. Sajad, "Ardebilipour Mehrdad. Multicast relay beamforming in CDMA networks: Nonregenerative approach", IEEE Communications Letters, vol. 19, no. 8, (2015), pp. 1418-1421.

[5] L. Tuyet V. Thi and K. Y. Hee, "Power and spectral efficiency of multi-pair massive antenna relaying systems with zero-forcing relay beamforming”, IEEE Communications Letters, vol. 19, no. 2, (2015), pp. 243-246.

[6] H. Wang, S. W. He and Z. H. Lu, "Robust Multi-cell Multi-user Cooperative Beamforming Technique Considering Transceiver Impairment and Imperfect Channel Information”, Journal of Electronics \& Information Technology, vol. 36, no. 5, (2014), pp. 1164-1170.

[7] T. Luo, H. W. Liu and J. K. Yan, "Robust Beamforming via Semidefinite Rank Relaxation”, Journal of Electronics \& Information Technology, vol. 36, no. 7, (2014), pp. 1545-1551.

[8] K. Zarifi, A. Ghrayeb and S. Affes, "Jointly optimal source power control and relay matrix design in multipoint-to-multipoint cooperative communication network", IEEE Transactions On Signal Processing, vol. 59, no. 9, (2011), pp. 4313-4330.

[9] D. Ponukumati, F. Gao and C. Xing, "Robust Peer-to-Peer Relay Beamforming: A Probabilistic Approach", IEEE Communications Letters, vol. 17, no. 2, (2013), pp. 305-308.

[10] W. C Li, T. H. Chang and C. Lin, "Coordinated Beamforming for Multiuser MISO Interference Channel Under Rate Outage Constraints”, IEEE Transactions On Signal Processing, vol. 61, no. 5, (2013), pp. $1087-1103$.

[11] D. Zheng, J. Liu, K. Wong and H. Chen, "Robust peer-to-peer collaborative-relay beamforming with ellipsoidal CSI uncertainties", IEEE Communications Letters, vol. 16, no. 4, (2012), pp. 442-443.

[12] S. H. Safavi, Mehrdad Ardebilipour and Soheil Salari, "Relay Beamforming in Cognitive Two-Way Networks with Imperfect Channel State Information", IEEE Wireless Communications Letters, vol. 1, no. 4, (2012), pp. 344-347.

[13] Y. Qin, M. Ding, M. Zhang, H. Yu and H. Luo, "Relaying Robust Beamforming for Device-to-Device Communication With Channel Uncertainty”, IEEE Communications Letters, vol. 18, no. 10, (2014), pp. $1859-1862$.

[14] S. F. Dehkordy, S. Shahbazpanahi and S. Gazor, "Multiple peer-to-peer communications using a network of relays", IEEE Transactions On Signal Processing, vol. 57, no. 8, (2009), pp. 3053-3062.

[15] Y. Huang, D. P. Palomar and S. Zhang, "Lorentz-Positive Maps and Quadratic Matrix Inequalities With Applications to Robust MISO Transmit Beamforming", IEEE Transactions On Signal Processing, vol. 61, no. 5, (2013), pp. 1121-1130. 


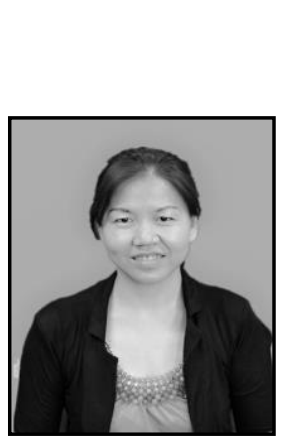

\section{Authors}

Jianhua Li, received her bachelor of science degree in mathematics from the Henan Normal University, Xinxiang, China, in 2002. She received her master of science degree in mathematics from the Zhengzhou University, Zhengzhou, China, in 2010. She is currently a lecturer at the College of Mathematical Science, Luoyang Normal University, China. Her research interests include communications technology and algorithm.

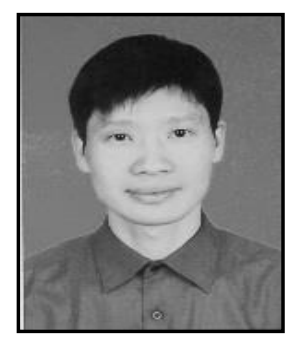

Yunshan Hou, received his bachelor of science degree in mathematics from the Zhengzhou University, Zhengzhou, China, in 1995. He received his master of science degree in computing from the National University of Singapore, Singapore, in 2004. $\mathrm{He}$ received Ph.D in Information engineering at Northwestern Polytechnical University, China, in 2011. He is currently an associate proffessor at the College of Information Engineering, Henan University of Science and Technology, China. His research interests include array signal processing and wireless communication. 